\title{
Brugada syndrome and p.E61X_RANGRF
}

\author{
Oscar Campuzano ${ }^{1 *}$, Paola Berne ${ }^{2 *}$, Elisabet Selga ${ }^{1}$, \\ Catarina Allegue ${ }^{1}$, Anna Iglesias ${ }^{1}$, Josep Brugada ${ }^{2}$, Ramon Brugada ${ }^{1}$ \\ ${ }^{1}$ Centre Genètica Cardiovascular, University of Girona-IdIBGi, Girona, Spain \\ ${ }^{2}$ Unitat Arrítmias, Hospital Clinic, Universitat de Barcelona, Barcelona, Spain
}

\begin{abstract}
Background: Brugada syndrome (BrS) is an inherited cardiac condition transmitted with an autosomal dominant pattern which can lead to sudden cardiac death from malignant ventricular arrhythmias. The RANGRF gene has recently been proposed to be associated with $B r S$. This gene encodes the MOG1 protein, a co-factor required for the full functioning of the cardiac sodium channel Nav1.5. The nonsense p.E61X genetic variation in the RANGRF gene has been postulated as responsible for BrS although no clear association has been established. Methods: We clinically and genetically evaluated a Spanish family diagnosed with BrS. A comprehensive genetic analysis of all genes to date responsible for BrS was performed in the index case.

Results: The index case was clinically diagnosed with BrS after flecainide test. We identified a nonsense variation ( $p . E 61 X)$ in the index case and in other 5 family members. All of them showed a normal electrocardiogram (ECG) in basal conditions. Flecainide test unmasked a type $1 \mathrm{BrS} E C G$ only in 2 of the relatives.

Conclusions: We suggest that p.E61X_RANGRF is a rare genetic variation with an uncertain role in BrS. Further studies must $\bar{b}$ e performed to elucidate the potential pathogenic role of p.E61X_RANGRF in BrS. (Cardiol J 2014; 21, 2: 121-127)
\end{abstract}

Key words: sudden cardiac death, Brugada syndrome, RANGRF gene, nonsense mutation

\section{Introduction}

Brugada syndrome $(\mathrm{BrS})$ is an inherited cardiac disease characterized by a typical electrocardiogram (ECG) pattern of ST segment elevation in the right precordial leads $\left(\mathrm{V}_{1}-\mathrm{V}_{3}\right)[1]$. BrS is associated with sudden cardiac death (SCD) by polymorphic ventricular tachycardia or ventricular fibrillation [2]. The prevalence of the disease is believed in the range of 5 to 50 cases per 10,000 individuals. However, it is difficult to ascertain because there is a high percentage of asymptomatic patients who remain undiagnosed and with a concealed ECG pattern [3-5]. The average age of onset of symptoms is about $30-40$ years, although they may occur both in childhood and in older age $[3,6]$. The diagnosis of $\mathrm{BrS}$ is based solely on clinical and ECG parameters [7], but actual criteria for a conclusive diagnosis have been highly debated [8-10].

$\mathrm{BrS}$ is a genetic disease [11], so far linked to SCN5A, GPD1L, CACNA1C, CACNB2, SCN1B, KCNE3, SCN3B, KCNJ8, HCN4, RANGRF, KCNE5, KCND3 and CACNA2D1 genes. These genes encode proteins that participate directly or

Address for correspondence: Ramon Brugada Terradellas, MD, PhD, FACC, FESC, Director Cardiovascular Genetics Center/Dean School of Medicine, Parc Cientific i Tecnologic, Universitat de Girona, 17003 Girona, Spain, e-mail: ramon@brugada.org

Received: 03.05.2013 Accepted: 28.08.2013

*Both authors equally contributed. 
indirectly in the generation of the cardiac action potential. Around $25 \%$ of $\mathrm{BrS}$ cases can be attributed to mutations identified in the SCN5A gene [12], which encodes the Nav1.5 protein. All other genes together are responsible for an additional $2-5 \%$ of total BrS cases [13-18]. Debate continues about BrS-related role of these minor genes because only a few mutations have been identified in few families. Therefore, further familiar genetic studies are required in additional $\mathrm{BrS}$ cohorts in order to validate the causative relationship between mutations in the less prevalent genes and BrS.

The RAN guanine nucleotide release factor $(R A N G R F)$ gene - Chr17p13.1 - has recently been associated to $\mathrm{BrS}$. This gene encodes the human MOG1 protein (Multicopy suppressor of Gsp1) [19] (human, Q9HD47 protein). To date, 4 isoforms due to alternative splicing (Q9HD47-1, Q9HD47-2, Q9HD47-3, Q9HD47-4) have been described; to date, Q9HD47-1 is considered the canonical sequence. MOG1 protein interacts with Nav1.5 channel via its intracellular loop between domains II and III, and co-localizes with the sodium channel at intercalated disks in ventricular myocytes [20]. Interestingly, Nav1.5 and MOG1 co-expression increases sodium current without changes in the channel biophysical properties, suggesting that MOG1 protein regulates the expression of Nav1.5 at the cell surface [21]. To date, only 2 variations in the RANGRF gene have been linked to BrS. The first described mutation was a missense variation (p.E83D) which impairs the trafficking of Nav1.5 to the membrane. This impairment reduces sodium current $\left(\mathrm{I}_{\mathrm{Na}}\right)$ and brings the phenotype of $\mathrm{BrS}$ [14]. However, the BrS phenotype showed by the patient carrying this genetic variation is still a matter of debate. The second genetic variation, p.E61X, was reported in an asymptomatic male patient with a type-1 BrS ECG pattern [22]. In vitro studies of this genetic variation also showed loss of $\mathrm{I}_{\mathrm{Na}}$ function. More recently, this nonsense variation was reported in a Danish cohort affected by lone atrial fibrillation (AF) [23]. The p.E61X genetic variation has therefore been associated with an increased risk of arrhythmias. However, the presence of this variation in $0.4 \%$ of the $\mathrm{Da}$ nish population [23] has brought into question its association with BrS [24].

In the present study, we performed a genotype-phenotype correlation in a Spanish family affected by BrS. Genetic screening identified p.E61X nonsense genetic variation in the RANGRF gene. Our goal was to clarify the potential pathogenic role of p.E61X_RANGRF in BrS.

\section{Methods}

\section{Clinical study}

Nine members of 1 Spanish family were clinically evaluated in Hospital Clinic (Barcelona, Spain). Informed consent of all the patients was obtained in accordance with the local institutional review board guidelines of Hospital Josep Trueta (Girona, Spain) and University of Girona (Girona, Spain). The diagnosis of BrS was accepted when the patients showed an ECG type 1 (basally or after the administration of intravenous sodium channel blockers) in conjunction with at least 1 clinical criterion, reflecting the occurrence of documented ventricular arrhythmia, family history (of SCD or $\mathrm{BrS}$ ) and/or symptoms secondary to arrhythmia. Structural heart disease was ruled out in all the participating individuals.

\section{Genetic analysis}

Genomic DNA was extracted from blood samples following commercial protocols (PUREGENE DNA, QIAGEN). Subsequently, polymerase chain reaction (PCR) was used to amplify each gene of interest. $\mathrm{PCR}$ products were purified using ExoSAP-IT (ISOGEN), and the analysis of the exonic and intron-exon regions was performed by direct sequencing (Genetic Analyzer 3130XL, Applied Biosystems). The proband underwent a genetic study of SCN5A (NM000335), GPD1L (NM015141), CACNA1C (NM000719), $C A C N B 2$ (NM000724), SCN1B (NM001037), KCNE3 (NM005472), SCN3B (NM018400), KCNE5 (NM012282), KCND3 (NM004980.4), KCNJ8 (NM004982), HCN4 (NM005477), CACNA2D1 (NM000722) and RANGRF (NM016492) genes (NCBI, National Center for Biotechnology Information, http://www.ncbi.nlm.nih.gov/). For each new genetic alteration identified, a genetic analysis was carried out in 328 Spanish unrelated control subjects (656 control alleles), individuals not related to any patient and of the same ethnicity. In silico pathogenicity of the genetic variation was consulted in CONDEL software (CONsensus DELeteriousness score of missense SNVs) (http:// bg.upf.edu/condel/). In addition, allelic frequency was consulted in Exome Variant Server (EVS) (http://evs.gs.washington.edu/EVS/).

\section{Results}

\section{Clinical characteristics}

The index case (II.3) was a 42-year-old male patient, who presented a type 2 BrS ECG pattern 


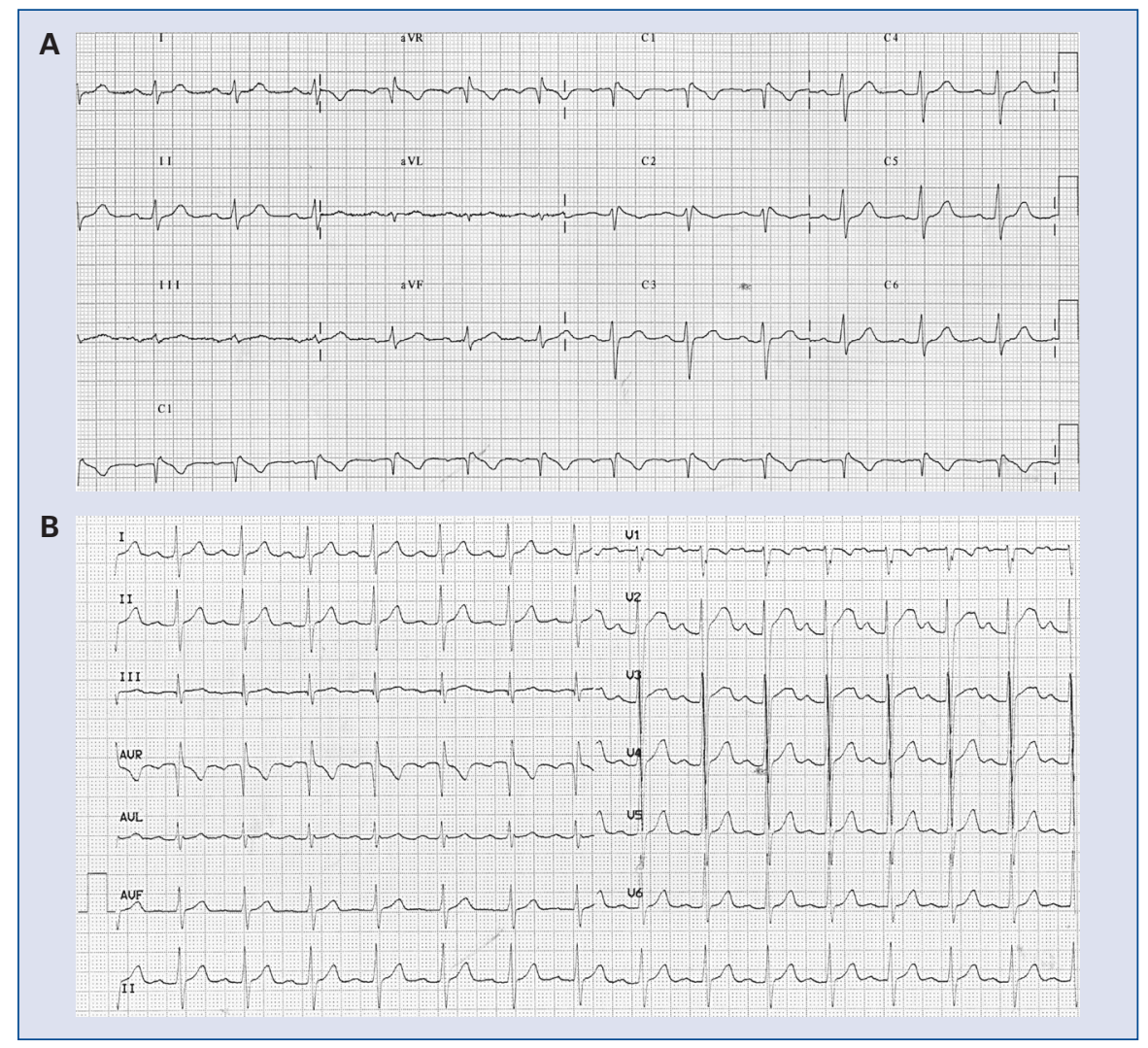

Figure 1. A. Twelve-lead electrocardiogram (ECG) of the proband (II.3) after flecainide test. The ECG shows a typical Brugada syndrome (BrS) pattern; B. Twelve-lead ECG of proband's son (III.1) after flecainide test. The ECG shows a negative BrS pattern.

during a routine medical evaluation at work. He had no previous history of syncope, seizures or nocturnal agonal respiration. He underwent a flecainide test, during which he developed a type 1 BrS pattern (Fig. 1A). Because 2 of his brothers (II.2 and II.7) had died suddenly after birth, he underwent electrophysiological study (EPS) for risk stratification. During EPS, ventricular fibrillation was induced, requiring external defibrillation. He received an implantable cardioverter defibrillator. None of his relatives showed a diagnostic BrS ECG pattern at baseline. Four relatives underwent flecainide test (II.1, II.5, II.6 and III.1). Two showed a positive BrS ECG (II.5, II.6) (Fig. 1B). Four family members did not undergo the flecainide test (I.1 73 years old, I. $2-71$ years old, II. $4-40$ years old, III.2 - 6 years old) (Fig. 2).

\section{Genetic analysis}

The index case (II.3) was screened for exons and flanking introns of all known BrS-related genes. A nonsense genetic variation (p.E61X) was identified in the RANGRF gene. A DNA sequencing

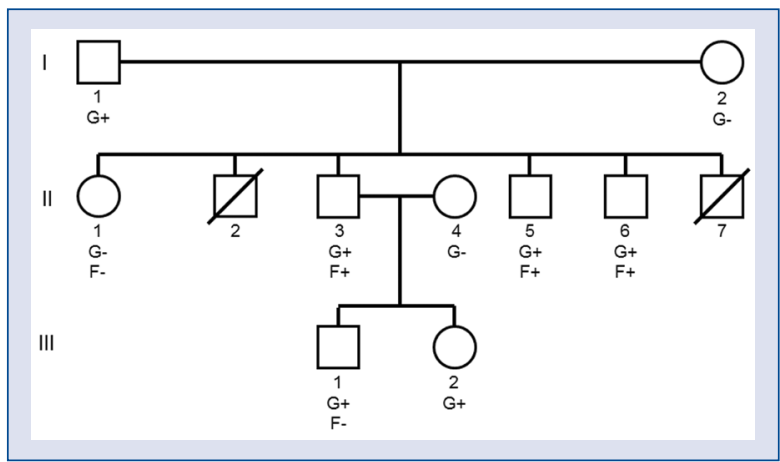

Figure 2. Pedigree. II.3 represents the index case. Squares represent men, circles represent women. Slashes represent deceased members. None of the patients displayed a diagnostic electrocardiogram type 1 pattern at baseline. Letter $\mathrm{G}$ with plus signs below circle/squares indicates carriers of the genetic variation p.E61X and letter $\mathrm{G}$ with minus signs below circle/squares indicates non-carriers. Letter $\mathrm{F}$ with plus signs below circle/squares indicates positive flecainide test. Letter $F$ with minus signs below circle/squares indicates negative flecainide test. No letter $\mathrm{F}$ below circle/squares indicates no flecainide test performed. 


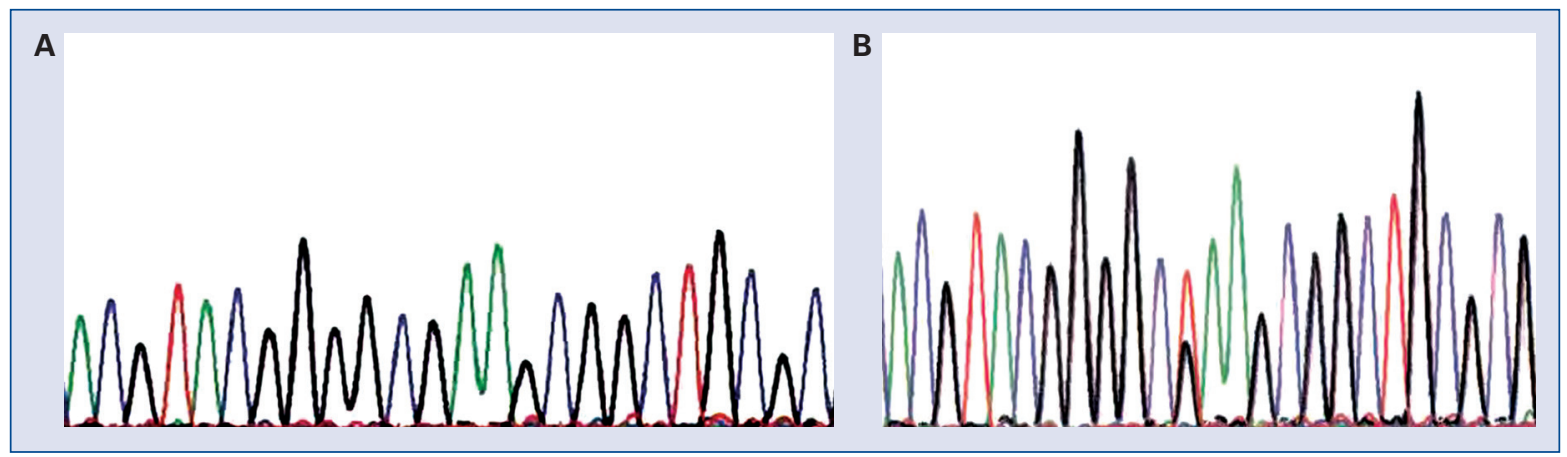

Figure 3. DNA forward sequence of the RANGRF gene. Normal sequence (A) and sequence with the genetic variation p.E61X (B). Note the substitution c.181G > T (p.Glu61OCH). The triplet GAA (E, Glu) is substituted by stop codon TAA $(\mathrm{X}, \mathrm{OCH})$.

analysis revealed a $\mathrm{G}$ to $\mathrm{T}$ base substitution at position 181 (c.181G $>$ T) in exon 2 which caused a change from GAA (E, Glu, glutamic acid) to stop codon TAA (X, OCH, OCHer) at position 61 (Fig. 3). This nonsense variation was previously reported, (rs140704891) although with unknown pathogenic effect. Three previously described single nucleotide polymorphisms (SNPs) were also identified, situated in 3 different genes (p.F66F in exon 2 of the KCNE3 gene - rs2270676; p.D600E in exon 13 of the CACNB2 gene - rs58225473; p.P1883P in exon 46 of the CACNA1C gene - rs56270948). The genetic evaluation of all family members confirmed that 5 of them carried the p.E61X genetic variation (I.1, II.5, II.6, III.1, and III.2) (Fig. 2).

\section{Phenotype-genotype correlation}

The index case (II.3) showed the BrS ECG pattern after flecainide test (Fig. 1) and carried the nonsense genetic variation (Fig. 3). Two of his brothers (II.5 and II.6) carried the same genetic variation, and flecainide test also revealed BrS ECG pattern in both of them (Fig. 2). The proband has 1 son (III.1) and 1 daughter (III.2), both carried the nonsense genetic variation. However, his son did not show BrS ECG pattern after flecainide test (Fig. 1B).

\section{In silico analysis}

The EVS database for the listed alleles in European-American (EA) showed an allele count of $\mathrm{T}=46 / \mathrm{G}=8550$. Therefore, the minor allele frequency (MAF) is $0.535 \%$. The EA genotype is $\mathrm{TT}=0 / \mathrm{TG}=46 / \mathrm{GG}=4252$. The NCBI database showed frequencies of $0.995 \%$ for the $\mathrm{G}$ allele and $0.005 \%$ for the T allele. Genotype distributions showed frequencies of $\mathrm{G} / \mathrm{G}: 0.989 \%, \mathrm{G} / \mathrm{T}: 0.011 \%$
Table 1. Alignment of the RANGRF gene isoforms in human. Bold $\mathbf{E}$ indicates the position of conserved amino acid between isoforms.

\begin{tabular}{lc}
\hline Aminoacid sequence & Gene Isoform \\
\hline ELLELQAHVRGEAAARYHFED & Q9HD47 \\
ELLELQAHVRGEAAARYHFED & Q9HD47-2 \\
ELLELQAHVRGEAAARYHFED & Q9HD47-3 \\
ELLELQAHVRGEAAARYHFED & Q9HD47-4 \\
\hline
\end{tabular}

and T/T: 0\%. The 1000 genomes database reports MAF $=0.2 \%$, in concordance with EVS database.

Evolutionary studies have described 4 different isoforms of human RANGRF (Q9HD47-1, 2, 3 and 4 ) produced by alternative splicing of this gene. Each isoform has a different protein length (186aa, 146aa, 165aa and 118aa, respectively) although all of them share the first region of $117 \mathrm{aa}$. The variation p.E61X is situated in the conserved region (Table 1). In addition, alignment studies show that this is a highly conserved region between different species (Table 2).

\section{Discussion}

We have performed a phenotype-genotype correlation in a Spanish family affected by BrS. The index case was clinically diagnosed with $\mathrm{BrS}$ after flecainide test, and genetic analysis revealed the presence of $\mathrm{p} . \mathrm{E} 61 \mathrm{X}$ genetic variation in the $R A N G R F$ gene. This genetic variation was previously reported by Kattygnarath et al. [22] in an asymptomatic patient with a type- 1 BrS ECG. This nonsense variation was again reported in a Danish cohort of patients with AF [23]. Finally, 
Table 2. Multiple sequence alignment. Conservation of the altered amino acid in the genetic variation $\mathrm{p} . \mathrm{E} 61 \mathrm{X}$. Bold $\mathbf{E}$ indicates the position of conserved amino acid between species.

\begin{tabular}{|c|c|}
\hline Aminoacid sequence & Specie \\
\hline ELLELQAHVRGEAAARYHFED & H. sapiens \\
\hline ELLELQAHVRGEAAARYHFED & C. lupus \\
\hline ELLELOAHVRGEAAARYHFED & B. taurus \\
\hline ELLELOAHVRGEAAARYHFED & R. norvegicus \\
\hline ELLELQAHVRGEAAARYHFED & Mus musculus \\
\hline ELLELQAOVRGEAAARYHFED & $\begin{array}{l}\text { Equus } \\
\text { caballus }\end{array}$ \\
\hline ELLELOAHVOGEAAARYHFED & $\begin{array}{l}\text { Canis } \\
\text { familiaris }\end{array}$ \\
\hline ELLELQAHVRGEAAARYHFED & $\begin{array}{l}\text { Oryctolagus } \\
\text { cuniculus }\end{array}$ \\
\hline ELLELQAHVRGEAAARYHFED & $\begin{array}{l}\text { Gorilla gorilla } \\
\text { gorilla }\end{array}$ \\
\hline
\end{tabular}

EPS studies in heterologous systems have shown that p.E61X abolishes Nav1.5 current [23]. All these data suggest a possible pathological causative role of this variation. However, it has been argued that the evidence provided in the BrS report is not sufficient for a conclusive diagnosis of $\mathrm{BrS}$ [24]. In addition, the variation has been identified in $0.4 \%$ of healthy individuals [23]. These have added to the ambiguity of the role of p.E61X_RANGRF in arrhythmogenesis. This phenomenon also occurs in other genetic variants of unknown significance (GVUS) because of recent exome studies in $\mathrm{BrS}$ cohorts suggesting no pathogenic role of several GVUS, previously associated with BrS [25]. This study suggests that exome data should be used as a tool in order to predict the pathogenicity of GVUS in patients suspected of BrS.

It was reported that different genetic mechanisms may explain the SCD cases [26]. Firstly, allelic imbalance has been identified in several human genes [27, 28], such as $S C N 5 A$ [29]. Thus, we cannot exclude that allelic expression imbalance involves a specific splice variant in our family, in concordance with Olesen et al. [24], who have recently suggested that the allelic imbalance could explain phenotype variability in p.E61X_RANGRF carriers. Therefore, one limitation of our study stems from the alternative splicing of RANGRF mRNA. Another mechanism responsible for inherited channelopathies is copy number variations, defined as a DNA segment of 1 kilobase $(\mathrm{kb})$ or larger which is present at a variable copy number in comparison to a reference genome, and that could cause a disease [30]. Thus, a recent study has identified a large-scale rearrangement of the $S C N 5 A$ gene supporting the association between the molecular pathology and the phenotypic expression in BrS [31]. Finally, the read-through mechanism could explain the phenotype variability in our family. It has also been suggested that potential damages from nonsense genetic variations may be reduced or even avoided to a tolerable level by translational read-through. This mechanism enables ribosomes to ignore the stop codon and produce full-length proteins. For instance, Teng et al. [32] reported the read-through of a nonsense mutation (p.W822X_SCN5A) that restored sodium channel expression.

In the present study we identified the genetic variation p.E61X_RANGRF in the index case and in 5 of his relatives. It is generally accepted that nonsense mutations, which lead to synthesis of a truncated proteins, should induce a severe effect in phenotype. In the case we are discussing, p.E61X RANGRF genetic variation induces a premature "stop codon" that truncates the protein upstream of the region that interacts with Nav1.5 [20]. Since protein MOG1 was described as a co-factor for optimal expression of Nav1.5 at cell membrane [21], p.E61X_RANGRF would potentially cause a disruption in Nav1.5 trafficking. In our study, none of the 6 family members that carried the genetic variation showed any symptoms or a BrS ECG pattern at baseline. However, 2 members of the family died suddenly at early ages as the first and only manifestation of $\mathrm{BrS}$, not infrequent in $\mathrm{BrS}$ families [33]. Unfortunately, we could not perform a genetic study in these deceased relatives. In the remaining relatives, flecainide test was performed to unmask a BrS phenotype. After flecainide test, 3 additional family members showed a BrS ECG pattern type 1 , and all of them carried the genetic nonsense variation. The $5^{\text {th }}$ family member, who carried the genetic variation, showed a negative flecainide test. The reason for that false negative result is not well explained in clinical practice, but it is well known that flecainide test does not unmask all BrS patients who carry a pathogenic mutation [34].

All these facts mentioned above are in accordance with incomplete penetrance and variable expressivity, hallmarks of $\mathrm{BrS}$ [35, 36]. Several reasons have been suggested to explain both features in $\mathrm{BrS}$, including gender, age and genetic factors [37]. The majority of affected patients in our family are males. The high proportion of males 
among BrS individuals suggests that male hormones may play a significant role in the phenotype. In addition, the average age of presentation of symptoms is approximately 40 years. Thus, comprehensive hormone studies could be performed in all family members to assess their role depending on gender and age. In addition, the disease could be related with epigenetic factors such as DNA methylation, post-translational modifications and RNA mechanisms [38, 39]. Finally, a combination of 2 or more genetic variations could be required to cause BrS phenotype in our family. Although we did not identify any other genetic variation, besides SNPs, in any of the other BrS tested genes, genetic variations in unknown genes may be responsible for the phenotype variability. While there is a clear link between the variation and the phenotype, the family is small, and therefore we cannot exclude the possibility that this co-segregation is just by coincidence. This fact is in concordance with the doubtful involvement of RANGRF in BrS, because the first genetic variant found in this gene (p.E83D_RANGRF) was identified in a patient with a non-conclusive BrS diagnostic [14, 24].

According to all information mentioned before, recent high-throughput genetics studies help to clarify the role of GVUS associated with SCD diseases, such as BrS. In addition, familial correlation is crucial to determine the potential pathogenicity of each one. So, despite recent genotype-phenotype studies, comprehensive clinical assessment is still the main current way to diagnose BrS patients.

\section{Conclusions}

We favor the hypothesis that p.E61X_RANGRF is a rare genetic variation associated with a mild BrS phenotype. The data suggest that the variation affects the sodium current sufficiently enough to unmask the BrS ECG under flecainide challenge, but not to bring the ECG pattern at baseline. Further studies must be performed in large $\mathrm{BrS}$ cohorts of different ethnic origin to elucidate the role of this nonsense genetic variation in $\mathrm{BrS}$.

\section{Acknowledgements}

The authors would like to thank Dr Pedro Beltran for his help in data interpretation and critical comments.

\section{Funding sources}

This work was supported by Centro Nacional de Investigaciones Cardiovasculares (CNIC-03-2008),
Instituto de Salud Carlos III (FIS-PI11/01826), and "Fundació La Caixa" for this study.

Conflict of interest: none declared

\section{References}

1. Brugada P, Brugada J. Right bundle branch block, persistent ST segment elevation and sudden cardiac death: A distinct clinical and electrocardiographic syndrome. A multicenter report. J Am Coll Cardiol, 1992; 20: 1391-1396.

2. Antzelevitch C, Brugada P, Brugada J et al. Brugada syndrome: A decade of progress. Circ Res, 2002; 91: 1114-1118.

3. Antzelevitch C, Brugada P, Borggrefe $\mathrm{M}$ et al. Brugada syndrome: Report of the second consensus conference: endorsed by the Heart Rhythm Society and the European Heart Rhythm Association. Circulation, 2005; 111: 659-670.

4. Antzelevitch C, Nof E. Brugada syndrome: recent advances and controversies. Curr Cardiol Rep, 2008; 10: 376-383.

5. Di Diego JM, Cordeiro JM, Goodrow RJ et al. Ionic and cellular basis for the predominance of the Brugada syndrome phenotype in males. Circulation, 2002; 106: 2004-2011.

6. Tester DJ, Ackerman MJ. Cardiomyopathic and channelopathic causes of sudden unexplained death in infants and children. Annu Rev Med, 2009; 60: 69-84.

7. Wilde AA, Antzelevitch C, Borggrefe M et al. Proposed diagnostic criteria for the Brugada syndrome: consensus report. Circulation, 2002; 106: 2514-2519.

8. Miyamoto A, Hayashi H, Makiyama T et al. Risk determinants in individuals with a spontaneous type 1 Brugada ECG. Circ J, 2011; 75: 844-851.

9. Kusano KF. ECG marker of high-risk in asymptomatic patients with Brugada syndrome. Circ J, 2011; 75: 777-778.

10. Lee LF, Felmlee N. Brugada syndrome: Unmasking a silent killer. Nursing, 2010; 40 (Suppl): 8-10.

11. Chen Q, Kirsch GE, Zhang D et al. Genetic basis and molecular mechanism for idiopathic ventricular fibrillation. Nature, 1998; 392: 293-296.

12. Kapplinger JD, Tester DJ, Alders $\mathrm{M}$ et al. An international compendium of mutations in the SCN5A-encoded cardiac sodium channel in patients referred for Brugada syndrome genetic testing. Heart Rhythm, 2010; 7: 33-46.

13. Ohno S, Zankov DP, Ding WG et al. KCNE5 (KCNE1L) variants are novel modulator of Brugada syndrome and idiopathic ventricular fibrillation. Circ Arrhythm Electrophysiol, 2011; 4: 352-361.

14. Kattygnarath D, Maugenre S, Neyroud N et al. MOG1: A new susceptibility gene for Brugada syndrome. Circ Cardiovasc Genet, 2011; 4: 261-268.

15. Giudicessi JR, Ye D, Tester DJ et al. Transient outward current (I(to)) gain-of-function mutations in the KCND3-encoded Kv4.3 potassium channel and Brugada syndrome. Heart Rhythm, 2011; 8: 1024-1032.

16. Haissaguerre M, Chatel S, Sacher F et al. Ventricular fibrillation with prominent early repolarization associated with a rare variant of KCNJ8/KATP channel. J Cardiovasc Electrophysiol, 2009; 20: 93-98.

17. Ueda K, Hirano Y, Higashiuesato Y et al. Role of HCN4 channel in preventing ventricular arrhythmia. J Hum Genet, 2009; 54: 115-121. 
18. Burashnikov E, Pfeiffer R, Barajas-Martinez $\mathrm{H}$ et al. Mutations in the cardiac L-type calcium channel associated with inherited J-wave syndromes and sudden cardiac death. Heart Rhythm, 2010; 7: 1872-1882.

19. Oki M, Nishimoto T. A protein required for nuclear-protein import, Mog1p, directly interacts with GTP-Gsp1p, the Saccharomyces cerevisiae ran homologue. Proc Natl Acad Sci USA, 1998; 95: 15388-15393.

20. Shy D, Gillet L, Abriel H. Cardiac sodium channel $\mathrm{Na}(\mathrm{V}) 1.5$ distribution in myocytes via interacting proteins: The multiple pool model. Biochim Biophys Acta, 2013; 1833: 886-894.

21. Wu L, Yong SL, Fan C et al. Identification of a new co-factor, MOG1, required for the full function of cardiac sodium channel Nav 1.5. J Biol Chem, 2008; 283: 6968-6978.

22. Kattygnarath D, Maugenre S, Neyroud N et al. MOG1 mutations associtaed with Brugada Syndrome electrocardiogram pattern. Circulation, 2009; 120: S686.

23. Olesen MS, Jensen NF, Holst AG et al. A novel nonsense variant in Nav1.5 cofactor MOG1 eliminates its sodium current increasing effect and may increase the risk of arrhythmias. Can J Cardiol; 2011; 27: 523.e17-23.

24. Olesen MS, Holst AG, Schmitt N. Letter by Olesen et al. regarding article, "MOG1: A new susceptibility gene for Brugada syndrome". Circ Cardiovasc Genet, 2011; 4: e22; author reply e23.

25. Risgaard B, Jabbari R, Refsgaard L et al. High prevalence of genetic variants previously associated with Brugada syndrome in new exome data. Clin Genet, 2013; 84: 489-495.

26. Banerjee B, Peiris DN, Koo SH et al. Genomic imbalances in key ion channel genes and telomere shortening in sudden cardiac death victims. Cytogenetic Gen Res, 2008; 122: 350-355.

27. Lo HS, Wang $Z$, Hu Y et al. Allelic variation in gene expression is common in the human genome. Genome Res, 2003; 13 : 1855-1862.

28. Yan H, Yuan W, Velculescu VE et al. Allelic variation in human gene expression. Science, 2002; 297: 1143.
29. Killen SA, Kunic J, Wang L et al. SCN5A allelic expression imbalance in African-Americans heterozygous for the common variant p.Ser1103Tyr. BMC Med Genet, 2010; 11: 74.

30. Redon R, Ishikawa S, Fitch KR et al. Global variation in copy number in the human genome. Nature, 2006; 444: 444-454.

31. Eastaugh LJ, James PA, Phelan DG, Davis AM. Brugada syndrome caused by a large deletion in SCN5A only detected by multiplex ligation-dependent probe amplification. J Cardiovasc Electrophysiol, 2011; 22: 1073-1076.

32. Teng S, Gao L, Paajanen V et al. Readthrough of nonsense mutation W822X in the SCN5A gene can effectively restore expression of cardiac Na+ channels. Cardiovasc Res, 2009; 83: 473-480.

33. Veerakul G, Nademanee K. Brugada syndrome. Circ J, 2012; 76: 2713-2722.

34. Meregalli PG, Ruijter JM, Hofman N et al. Diagnostic value of flecainide testing in unmasking SCN5A-related Brugada syndrome. J Cardiovasc Electrophysiol, 2006; 17: 857-864.

35. Probst V, Allouis M, Sacher F et al. Progressive cardiac conduction defect is the prevailing phenotype in carriers of a Brugada syndrome SCN5A mutation. J Cardiovasc Electrophysiol, 2006; 17: 270-275.

36. Giudicessi JR, Ackerman MJ. Determinants of incomplete penetrance and variable expressivity in heritable cardiac arrhythmia syndromes. Translational Res: J Lab Clin Med, 2013; 161: 1-14.

37. Probst V, Veltmann C, Eckardt L et al. Long-term prognosis of patients diagnosed with Brugada syndrome: Results from the FINGER Brugada Syndrome Registry. Circulation, 2011; 121: 635-643.

38. Webster AL, Yan MS, Marsden PA. Epigenetics and cardiovascular disease. Can J Cardiol, 2013; 29: 46-57.

39. Kim GH, Ryan JJ, Archer SL. The role of redox signaling in epigenetics and cardiovascular disease. Antioxidants Redox Sig, 2013; 18: 1920-1936. 